\title{
Intensidad del Punto de Contacto en Resinas Clase II. Revisión Sistemática
}

\author{
Proximal Contact Tightness in Class II Resins. Systematic Review
}

\author{
Javiera Sobarzo'; Tamara Vargas'; Patricio Ruiz² \& Isabel Riquelme²
}

SOBARZO, J.; VARGAS, T.; RUIZ, P. \& RIQUELME, I. Intensidad del punto de contacto en resinas clase II. Revisión sistemática. Int. J. Odontostomat. 15(4):882-887, 2021.

RESUMEN: Factores como el tipo de resina compuesta y sistema de matrices son clave para lograr una adecuada intensidad del punto contacto, la cual puede determinar el éxito de una restauración clase II. Con el objetivo de comparar la intensidad del punto de contacto obtenida en restauraciones de resina clase II, cuando se usan diferentes consistencias de resinas compuestas y sistemas de matrices en estudios in vitro, se realizó una revisión sistemática según la pauta PRISMA. Los estudios fueron seleccionados a través de una estrategia de búsqueda para cada base de datos electrónica (MEDLINE/ PubMed, Scopus, Web of Science y EBSCO host). En cada artículo seleccionado, se tabularon: autor/año, tamaño muestral, diente utilizado y preparación cavitaria, tipo de resina compuesta, tipo de sistema matriz e intensidad del punto de contacto (resultado medido en Newton). La calidad metodológica se analizó mediante una pauta modificada para estudios in vitro. De 90 artículos, un total de 5 artículos reunieron los criterios de selección y fueron incluidos. De estos cinco artículos, sólo dos artículos estudiaron la influencia de la consistencia de la resina compuesta y los sistemas de matrices en forma simultánea. El riesgo de sesgo para los estudios fue bajo para 1 artículo, medio para 2 y alto para los otros 2 artículos. No se obtiene la misma intensidad de punto de contacto entre diferentes consistencias de resina compuesta y distintos sistemas de matrices, ya que los mayores valores se obtuvieron al utilizar matrices seccionales combinadas con anillo de separación y resinas compuestas de mayor viscosidad.

PALABRAS CLAVE: composite, resina, resina compuesta, intensidad de contacto proximal, fuerza de contacto proximal.

\section{INTRODUCCIÓN}

Lograr una adecuada intensidad del punto de contacto proximal en restauraciones clase II representa un desafío en la práctica clínica. En este sentido, una buena restauración es el producto de tres factores fundamentales: una correcta indicación basada en un buen diagnóstico, óptima técnica operatoria y adecuada selección y manejo del material a utilizar (Shannon, 2006). El papel fisiológico que desempeña el punto de contacto, y la intensidad de este, es crucial, ya que su deficiencia contribuye a la impactación de alimento, caries recurrente, migración dentaria, movilidad y complicaciones periodontales (Kornman \& Löe, 1993). La intensidad del punto de contacto (IPC) se ha estudia- do en relación con diferentes sistemas de matrices, métodos de separación, tipos de resinas compuestas e intensidad de luz de fotocurado (El-Shamy et al., 2019), hallándose en modelos in vitro, que el sistema de matriz circunferencial usado en combinación con resina compuesta de alta viscosidad produce contactos proximales más intensos que cuando se utiliza resina compuesta de viscosidad media. Diferencia que no se observa cuando se utiliza un sistema de matriz seccional (Peumans et al., 2001). Debido a esto, la elección de una resina compuesta y un sistema de matriz son clínicamente relevantes para obtener un punto de contacto proximal funcional, de intensidad

\footnotetext{
${ }^{1}$ Escuela de Odontología, Facultad de Medicina, Universidad Austral de Chile, Valdivia, Chile.

2 Instituto de Odontoestomatología, Facultad de Medicina, Universidad Austral de Chile, Valdivia, Chile.
}

Recibido: 2021-05-26 Aceptado: 2021-08-27 
adecuada, disminuir el tiempo operatorio y conseguir resultados clínicos exitosos. Por lo tanto, el objetivo de esta revisión sistemática es comparar la IPC obtenida en restauraciones de resina clase II, cuando se usan diferentes consistencias de resinas compuestas y sistemas de matrices, en estudios in vitro.

\section{MATERIAL Y MÉTODO}

La revisión sistemática se realizó según la pauta PRISMA (Urrútia \& Bonfill, 2010). Los estudios fueron seleccionados a través de una estrategia de búsqueda para cada base de datos electrónica (MEDLINE/ PubMed, Scopus, Web of Science y EBSCO host) (Tabla I). La búsqueda y selección de estudios fue realizada entre el 26 y 30 de mayo del año 2020 y no se consideró ningún tipo de filtro de búsqueda.

Los artículos fueron evaluados por dos revisores (J.S. y T.V.) de forma independiente a través de lectura de título y abstract para la selección inicial. Los criterios de inclusión fueron: primeros molares de marfilina donde se realizaron restauraciones de resina compuesta de 2 superficies, estudios in vitro con resultados obtenidos a través de un medidor de presión en el área de contacto proximal, expresados en Newton (N). Los criterios de exclusión fueron: dientes humanos y animales, estudios descriptivos, reportes de casos, revisiones sistemáticas y estudios con otros materiales diferentes a resina compuesta. Luego, mediante la lectura del texto completo, se aplicaron los criterios de inclusión y exclusión para la selección final. En los casos que existió desacuerdo en la elegibilidad de algún artículo, este fue resuelto por un tercer y cuarto revisor (P.R. e I.R.).
En cada artículo seleccionado, se tabularon los datos: autor, año, tamaño de la muestra, diente utilizado y preparación cavitaria, tipo de resina compuesta, tipo de sistema de matriz e IPC (resultado medido en N) (Tabla II). Para evaluar el riesgo de sesgo de los artículos se adaptó una pauta modificada para estudios in vitro basada en la revisión sistemática de Gerula-Szymanska et al. (2020) (Tabla III), donde se asigna un punto si el parámetro está presente y no se asigna puntaje cuando los datos no son específicos. Estos fueron clasificados en riesgo de sesgo alto (1-3 puntos), medio (4-5 puntos) y bajo (6-8 puntos).

\section{RESULTADOS}

Un total de 90 artículos fueron encontrados en las 4 bases de datos electrónicas seleccionadas. En la Figura 1 se presenta el diagrama de flujo que muestra el proceso de selección de artículos según la pauta PRISMA. Finalmente, fueron incluidos 5 artículos que reunieron los criterios de selección. Estos estudios y los datos recogidos se muestran en la Tabla II.

De los artículos seleccionados, sólo dos (Loomans et al., 2006a; El-Shamy et al., 2019) estudiaron la influencia de la consistencia de la resina compuesta y los sistemas de matrices en forma simultánea.

El riesgo de sesgo para los estudios fue bajo para el artículo de El-Shamy et al. (2019), dos de riesgo medio (Saber et al., 2010; El-Shamy et al., 2012) y dos de riesgo alto (Loomans et al., 2006a,b); estando ausente la explicación del cálculo del tamaño muestral, grupo control, aleatorización y cegamiento del examinador en la mayoría de los artículos (Tabla III).

Tabla I. Estrategia de búsqueda realizada en bases de datos.

\begin{tabular}{|c|c|}
\hline Base de datos & Estrategia de búsqueda \\
\hline $\begin{array}{l}\text { Medline } \\
\text { (Pubmed) }\end{array}$ & $\begin{array}{l}\text { ((“composite"[TIAB] OR "resin"[TIAB] OR "dental composite resin"[TIAB] OR "composite } \\
\text { resin"[TIAB]) AND ("proximal contact tightness"[TIAB] OR "proximal contact } \\
\text { strength"[TIAB])) }\end{array}$ \\
\hline Web of Science & $\begin{array}{l}\text { ((TS=("composite") OR TS=("resin") OR TS=("dental composite resin") OR } \\
\text { TS }=(\text { composite resin)) AND (TS=("proximal contact tightness") OR TS=("proximal } \\
\text { contact strength") OR TS=("tightness of proximal tooth contact"))) }\end{array}$ \\
\hline Scopus & $\begin{array}{l}\text { ((TITLE-ABS-KEY (“composite") OR TITLE-ABS-KEY (“resin”) OR TITLE-ABS-KEY } \\
\text { (“dental composite resin”) OR TITLE-ABS-KEY (“composite resin")) AND (TITLE-ABS- } \\
\text { KEY ("proximal contact tightness") OR TITLE-ABS-KEY (“proximal contact strength") OR } \\
\text { TITLE-ABS-KEY("tightness of proximal tooth contact”))) }\end{array}$ \\
\hline EBSCO host & $\begin{array}{l}\text { ((TX “composite" OR TX "resin" OR TX "dental composite resin" OR TX "composite } \\
\text { resin") AND (TX "proximal contact tightness" OR TX "proximal contact strength" OR TX } \\
\text { "tightness of proximal tooth contact")) }\end{array}$ \\
\hline
\end{tabular}




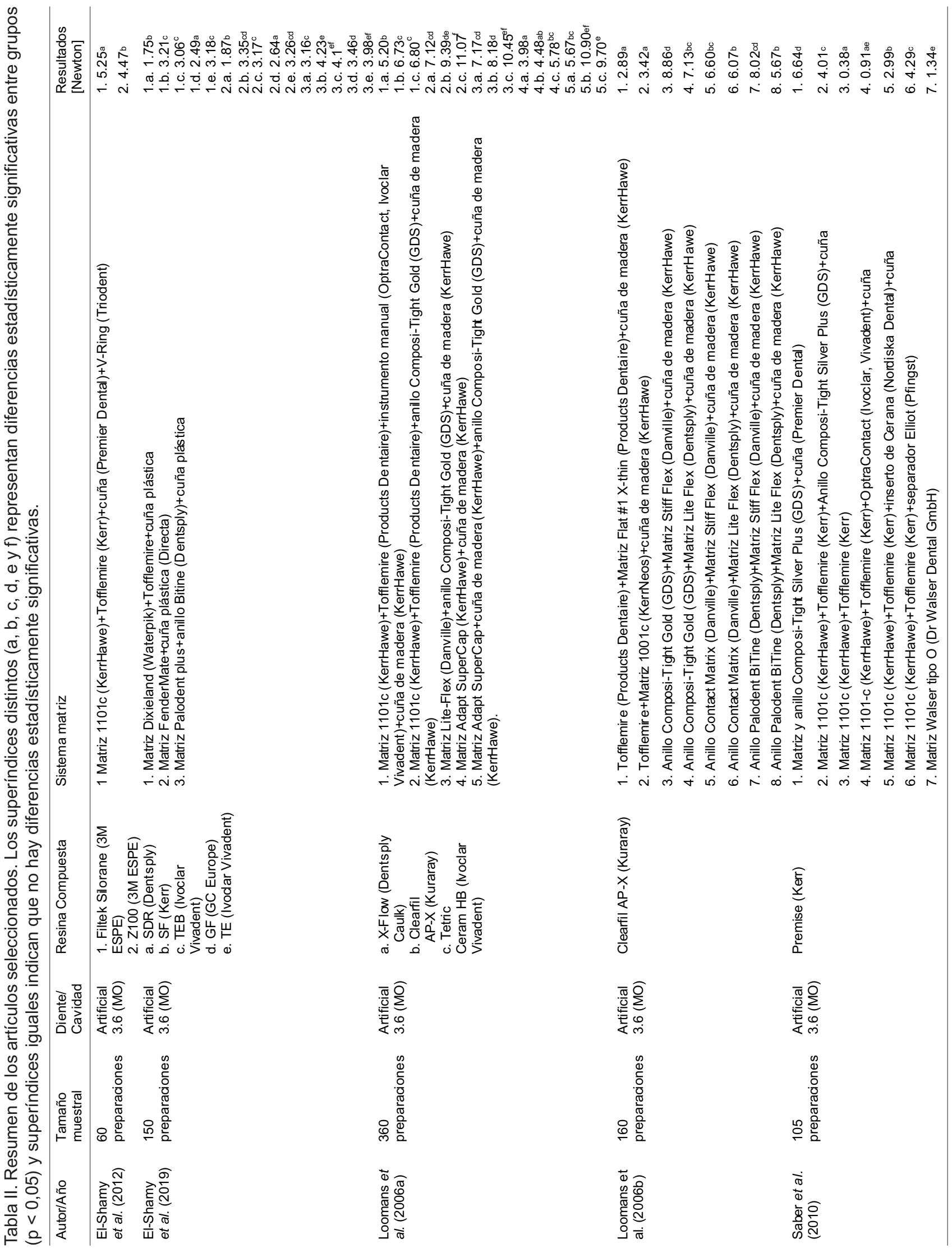




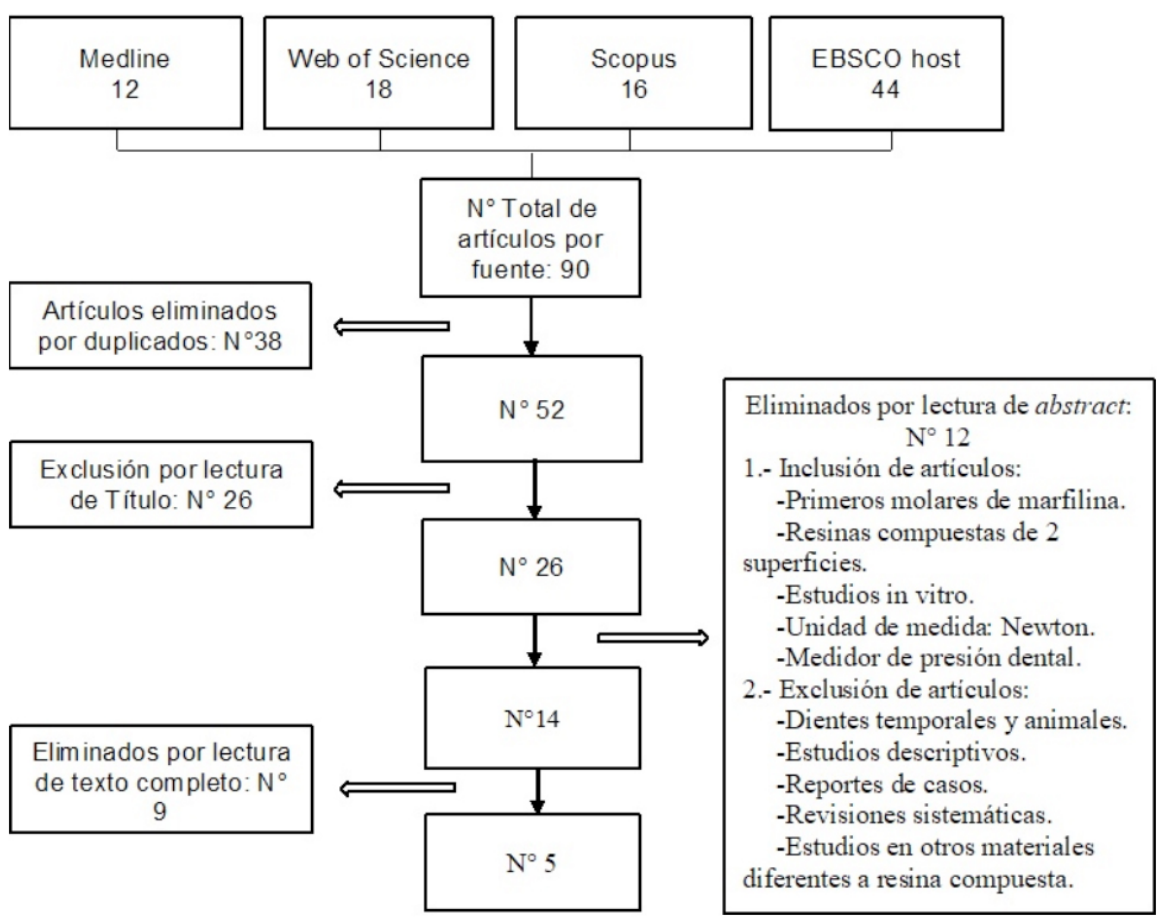

Fig. 1. Diagrama de flujo de búsqueda de acuerdo con la pauta PRISMA.

Tabla III. Evaluación de riesgo de sesgo en estudios in vitro. Puntaje: 1-3= riesgo de sesgo alto; 4-5= riesgo de sesgo medio; $6-8=$ riesgo de sesgo bajo.

\begin{tabular}{|c|c|c|c|c|c|}
\hline Preguntas escala estudios in vitro* & $\begin{array}{l}\text { El-Shamy } \\
\text { et al. (2012) }\end{array}$ & $\begin{array}{l}\text { El-Shamy et } \\
\text { al. (2019) }\end{array}$ & $\begin{array}{l}\text { Loomans et } \\
\text { al. (2006a) }\end{array}$ & $\begin{array}{l}\text { Loomans et } \\
\text { al. (2006b) }\end{array}$ & $\begin{array}{l}\text { Saber et } \\
\text { al. }(2010)\end{array}$ \\
\hline ¿Se utiliza el mismo tipo de diente? & 1 & 1 & 1 & 1 & 1 \\
\hline ¿Explica el cálculo muestral? & 0 & 0 & 0 & 0 & 0 \\
\hline ¿Utiliza tamaño similar de muestra? & 1 & 1 & 1 & 1 & 1 \\
\hline ¿Utiliza grupo control? & 0 & 1 & 0 & 0 & 1 \\
\hline ¿Se aleatorizan las muestras? & 0 & 1 & 0 & 0 & 0 \\
\hline ¿Se utilizan cavidades y mediciones estandarizadas? & 1 & 1 & 1 & 1 & 1 \\
\hline ¿Se utilizan los materiales según instrucciones del fabricante? & 1 & 1 & 0 & 0 & 1 \\
\hline ¿Existe cegamiento del examinador? & 1 & 1 & 0 & 0 & 0 \\
\hline Puntaje Total & 5 & 7 & 3 & 3 & 5 \\
\hline
\end{tabular}

\section{DISCUSIÓN}

La consistencia de las resinas compuestas depende de la fracción de volumen de relleno, el tipo de relleno y su distribución (García et al., 2006); y esto determina, en conjunto con factores como la dinámica de la polimerización y grado de conversión, la contracción volumétrica que sufre una resina compuesta (Salem et al., 2019). En el caso de las resinas compuestas basadas en monómeros de metacrilato, las moléculas de monómeros se encuentran débilmente unidas por fuerzas de Van der Waals que luego de la polimerización se transforman en uniones estrechas a través de enlaces covalentes, por lo que ocurre la contracción de polimerización (Kaisarly \& El-Gezawi, 2016). A diferencia de las resinas compuestas basadas en siloranos, los cuales corresponden a polímeros epóxicos que polimerizan a través de apertura de anillos que les otorgan una menor contracción de polimerización (Dörfer et al., 2001; Duarte et al., 2009), lo cual puede explicar los resultados obtenidos por El-Shamy et al. (2012). Sin embargo, los autores explican que las diferencias encontradas también podrían ser atribuidas a las distintas consistencias de resinas compuestas uti- 
lizadas y al manejo de ellas, así como a la falta de ciego del operador, puesto que este conocía el material que manipuló.

La clasificación de las resinas compuestas se realiza según la composición del relleno, matriz monomérica, viscosidad y clasificación del fabricante. De acuerdo a esta última, han aparecido en el mercado las resinas compuestas bulk-fill, presentes en dos consistencias (fluidas y empacables), al igual que las resinas compuestas convencionales (Schwendicke et al., 2018). Esta clasificación es dependiente de la fracción de volumen de relleno, por lo que las resinas compuestas bulk-fill presentan una contracción volumétrica que es dependiente de su cantidad de relleno, y este parece ser un factor más importante que la condición de ser bulk-fill. El-Shamy et al. (2019) no encontraron diferencias estadísticamente significativas en la IPC entre resinas compuestas bulk-fill de mayor contenido de relleno, y una resina compuesta convencional con alto contenido de relleno, pero sí se hallaron entre resinas compuestas fluidas (bulk-fill y convencionales) y resinas compuestas de mayor consistencia (bulk-fill y convencionales). Las resinas compuestas bulk-fill según su fabricante presentan una mayor profundidad de curado, mayor grado de conversión y menor contracción de polimerización que las resinas compuestas convencionales (Makhdoom et al., 2020); no obstante esto, resinas compuestas de este tipo con alta consistencia como SonicFill (Kerr, Orange, CA, USA) y Tetric Evoceram Bulkfill (IvoclarVivadent, Amherst, NY, USA), con porcentajes de relleno $83,5 \%$ y $80 \%$ respectivamente, son similares al porcentaje de relleno de Tetric EvoCeram (IvoclarVivadent), la cual es una resina compuesta convencional, que es de $82,5 \%$. Por otro lado, las resinas compuestas de consistencia fluida ya sean convencionales o bulk-fill, contienen menores porcentajes de relleno y no presentan diferencias estadísticamente significativas en la IPC entre ellas (El-Shamy et al., 2019); aunque, se atribuyen las diferencias encontradas a la consistencia y características de manipulación de las resinas compuestas estudiadas, ya que una resina compuesta fluida es de mayor dificultad de manejo que una de mayor consistencia. Además, estos autores no encontraron diferencias entre las resinas compuestas fluidas (bulk-fill y convencionales), sin embargo, la resina SDR (Dentsply, Milford, MA, USA) fue utilizada sin cobertura de resina compuesta convencional como lo recomienda su fabricante (Roggendorf et al., 2011), lo que puede haber influenciado en los resultados. Del mismo modo Loomans et al. (2006a), obtuvo los menores valores de la intensidad del punto de contacto en resinas compuestas de baja viscosidad (fluidas). Las resinas compuestas fluidas presentan mayor contracción de polimerización, por lo cual se separan de la banda matriz en mayor magnitud en restauraciones clase II, ya que la resina compuesta fluida no se adhiere a la banda matriz, y por consiguiente presentan una menor IPC (Kampouropoulos et al., 2010).

Otros factores que se han estudiado en relación a la IPC, es el uso de diferentes sistemas de matrices, cuñas y anillos de separación; no obstante, todos los estudios seleccionados para esta revisión determinaron que el mayor efecto en la IPC lo otorga la utilización de anillos de separación. El-Shamy et al. (2019) encontraron que el uso de anillos de separación genera diferencias estadísticamente significativas en la IPC, independiente del tipo de resina compuesta utilizada; lo que podría estar relacionado a la generación de presión constante y la consecuente separación interdentaria producida por los brazos de los anillos sobre el contacto proximal; por otra parte, las cuñas no tenían ningún efecto en la separación. Se ha evaluado el grado de separación comparando el uso del acuñamiento previo con el uso de anillo de separación, encontrándose que el efecto de la cuña es mínimo en la separación y que el mayor efecto se logra con el uso de anillos. Por lo que la función de las cuñas se limitaría a asegurar la adaptación de la matriz contra el diente y mantener el campo libre de la penetración de humedad (Loomans et al., 2007). Por otro lado, Loomans et al. (2006a) no encontró diferencias significativas en la IPC cuando se compararon matrices circunferenciales y seccionales al utilizar anillos de separación en ambas. Por lo tanto, un factor importante es lograr la separación proximal para compensar la contracción de polimerización y el grosor de la banda matriz. En el otro artículo de Loomans et al. (2006b) se encontró diferencias estadísticamente significativas en la IPC cuando compararon el uso de matrices circunferenciales con matrices seccionales combinadas con anillos de separación, pues en todos los grupos en los que se utilizaron anillos de separación se obtuvo mayor IPC que en los grupos en los que no se utilizó. Al igual que en Saber et al., los mayores valores de IPC estaban en los grupos con matrices seccionales combinados con anillos de separación.

Finalmente, se concluye que no se obtiene la misma IPC entre diferentes consistencias de resina compuesta y distintos sistemas de matrices, ya que los mayores valores se obtienen al utilizar matrices seccionales combinadas con anillo de separación y resinas compuestas de mayor viscosidad. Aunque parece ser que influiría más la separación interdental proporcionada por los anillos. Sin embargo, a pesar de lo anterior, la mayor 
parte de los artículos presentan riesgo de sesgo alto y medio, por lo que, se requieren mayor cantidad de estudios con buena calidad metodológica en el futuro.

SOBARZO, J.; VARGAS, T.; RUIZ, P. \& RIQUELME, I. Proximal contact tightness in class II resins. Systematic review. Int. J. Odontostomat. 15(4):882-887, 2021.

ABSTRACT: Factors such as the type of composite resin and matrix system are key to achieving an adequate proximal contact tightness, which can determine the success of a class II restoration. To compare the proximal contact tightness obtained in class II resin restorations, when different consistencies of composite resins and matrix systems are used, on in vitro studies. The systematic review was carried out according to the PRISMA Statement guidelines. The studies were selected through a search strategy for each electronic database (MEDLINE/PubMed, Scopus, Web of Science and EBSCO host). In each selected article, the following were tabulated: author/year, sample size, tooth used and cavity preparation, type of composite resin, type of matrix system and proximal contact tightness (result measured in Newton). The methodological quality was analyzed using a modified guideline for in vitro studies. Of 90 articles, a total of 5 articles met the selection criteria and were included. Of these five articles, only two articles studied the influence of the consistency of the composite resin and the matrix systems simultaneously. One of the articles was a low risk of bias, two of medium and two of high. The same proximal contact tightness is not obtained between different consistencies of composite resin and different matrix systems, since the highest values were obtained when using sectional matrices combined with a separation ring and composite resins of higher viscosity.

KEY WORDS: composite, resin, composite resin, proximal contact tightness, proximal contact strength.

\section{REFERENCIAS BIBLIOGRÁFICAS}

Dörfer, C; Schriever, A.; Heidemann, D.; Staehle, H. \& Pioch, T. Influence of rubber-dam on the reconstruction of proximal contacts with adhesive tooth-colored restorations. J. Adhes. Dent., 3(2):169-75, 2001.

Duarte Jr., S.; Botta, A. C.; Phark, J. H. \& Sadan, A. Selected mechanical and physical properties and clinical application of a new low-shrinkage composite restoration. Quintessence Int., 40(8):631-8, 2009.

El-Shamy, H.; Saber, M. H.; D?rfer, C. E.; El-Badrawy, W. \& Loomans, B. A. C. Influence of volumetric shrinkage and curing light intensity on proximal contact tightness of class II resin composite restorations: in vitro study. Oper. Dent., 37(2):205-10, 2012.

El-Shamy, H.; Sonbu, H.; Alturkestani, N.; Tashkandi, A.; Loomans, B.; Dörfer, C. \& El-Badrawy, W. Proximal contact tightness of class ? bulk-fill composite resin restorations: An in vitro study. Dent. Mater. J., 38(1):96-100, 2019.

García, A.; Martínez, M.; Cabanes, J.; Barjau, A. \& Fos, P. Resinas compuestas. Revisión de los materiales e indicaciones clínicas. Med. Oral Patol. Oral Cir. Bucal, 11(2):215-20, 2006.
Gerula-Szyman'ska, A.; Kaczor, K.; Lewusz-Butkiewicz, K. \& Nowicka, A. Marginal integrity of flowable and packable bulk fll materials used for class II restorations-A systematic review and meta-analysis of in vitro studies. Dent. Mater. J., 39(3):335-44, 2020.

Kaisarly, D. \& El-Gezawi, M. Polymerization shrinkage assessment of dental resin composites: a literature review. Odontology, 104(3):257-70, 2016.

Kampouropoulos, D.; Paximada, C.; Loukidis, M. \& Kakaboura, A. The influence of matrix type on the proximal contact in class II resin composite restorations. Oper. Dent., 35(4):454-62, 2010.

Kornman, K. S. \& Löe, H. The role of local factors in the etiology of periodontal diseases. Periodontol. 2000, 2(1):83-97, 1993.

Loomans, B.; Opdam, N.; Bronkhorst, E.; Roeters, F. \& Dörfer, C. A clinical study on interdental separation techniques. Oper. Dent., 32(3):207-11, 2007.

Loomans, B.; Opdam, N.; Roeters, F.; Bronkhorst, E. \& Burgersdijk, R. Comparison of proximal contacts of Class II resin composite restorations in vitro. Oper. Dent., 31(6):688-93, 2006b.

Loomans, B.; Opdam, N.; Roeters, J.; Bronkhorst, E. \& Plasschaert, A. Influence of composite resin consistency and placement technique on proximal contact tightness of Class II restorations. J. Adhes. Dent., 8(5):305-10, 2006a.

Makhdoom, S. N.; Campbell, K. M.; Carvalho, R. M. \& Manso, A. P. Effects of curing modes on depth of cure and microtensile bond strength of bulk fill composites to dentin. J. Appl. Oral Sci., 28:e20190753, 2020.

Peumans, M.; Van Meerbeek, B.; Asscherickx, K.; Simon, S.; Abe, Y.; Lambrechts, P. \& Vanherle, G. Do condensable composites help to achieve better proximal contacts? Dent. Mater., 17(6):53341, 2001.

Roggendorf, M.; Krämer, N.; Appelt, A.; Naumann, M. \& Frankenberger, R. Marginal quality of flowable 4-mm base vs. Conventionally layered resin composite. J. Dent., 39(10):643-7, 2011.

Saber, M.; Loomans, B.; El-Zohairy, A.; D?rfer, C. \& El-Badrawy, W. Evaluation of proximal contact tightness of Class II resin composite restorations. Oper. Dent., 35(1):37-43, 2010.

Salem, H.; Hefnawy, S. \& Nagi, S. Degree of conversion and polymerization shrinkage of low shrinkage bulk-fill resin composites. Contemp. Clin. Dent., 10(3):465-70, 2019.

Schwendicke, F.; Blunck, U.; Tu, Y. K. \& Göstemeyer, G. Does classification of composites for network meta-analyses lead to erroneous conclusions? Oper. Dent., 43(2):213-22, 2018.

Shannon, A. T. Achieving form and function for Class II restorations using aesthetic resin stratification. Pract. Proced. Aesthet. Dent., 18(5):323-8, 2006.

Urrútia, G. \& Bonfill, X. Declaración PRISMA: una propuesta para mejorar la publicación de revisiones sistemáticas y metaanálisis. Med. Clin., 135(11):507-11, 2010.

Dirección para correspondencia:

Javiera Sobarzo

Escuela de Odontología

Facultad de Medicina

Universidad Austral de Chile

Valdivia

CHILE

E-mail: javiitandrea@gmail.com 